\title{
An Optimal Surface Concentration of Pure Cardiolipin Deposited Onto Glassy Carbon Electrode Promoting the Direct Electron Transfer of Cytochrome-c
}

Estelle Lebègue ${ }^{a}{ }^{*}$ Hassiba Smida, ${ }^{a}$ Thomas Flinois, ${ }^{a}$ Véronique Vié,${ }^{b}$ Corinne Lagrost, ${ }^{a}$ and Frédéric Barrière $^{a *}$

*corresponding authors: frederic.barriere@univ-rennes1.fr and estelle.lebegue@univrennes1.fr

${ }^{a}$ Institut des Sciences Chimiques de Rennes, Equipe MaCSE, UMR CNRS 6226, Université de Rennes 1, Campus de Beaulieu 35042 Rennes, France

${ }^{b}$ Institut de Physique de Rennes, UMR CNRS 6251, Université de Rennes 1, Campus de Beaulieu 35042 Rennes, France

\begin{abstract}
Pure cardiolipin deposit onto electrodes is optimized and shown to yield an efficient supported lipid film for promoting cytochrome-c immobilization and electroactivity. Cyclic voltammetry and electrochemical impedance spectroscopy measurements in an aqueous electrolyte with potassium ferri- and ferrocyanide as a redox probe evidence that an optimized pure cardiolipin film is reached for a $7 \mu \mathrm{g} \mathrm{cm}^{-2}$ deposit onto glassy carbon electrode. At this optimized surface concentration the pure cardiolipin deposit yields the most compact and less permeable supported lipid film on electrode surface. The thickness and the organization of the pure cardiolipin films were analyzed by atomic force microscopy (AFM) measurements. AFM imaging in aqueous buffer shows that the lipid deposit onto the surface forms a thick deposit of approximately $30 \pm 10 \mathrm{~nm}$ of height with $4 \mathrm{~nm}$ average roughness and includes
\end{abstract}


defects. Cytochrome- $c$ electroactivity was studied with the redox protein either in solution or immobilized onto the modified electrode. First, the optimized amount of pure cardiolipin was deposited onto glassy carbon electrodes to study the stable and electrochemically quasireversible redox system of cytochrome-c in solution. Then, the potential cycling of a pure cardiolipin-modified glassy carbon electrode in a cytochrome-c solution led to the immobilization of the protein in its native state keeping intact its electrochemical properties, and with a surface coverage of $8 \mathrm{pmol} \mathrm{cm}^{-2}$ corresponding to $50 \%$ of a monolayer.

Keywords. Cardiolipin, Cytochrome-c, Supported Lipid Deposit, Cyclic Voltammetry, Electrochemical Impedance Spectroscopy, Atomic Force Microscopy. 


\section{Introduction}

Cardiolipin is a phospholipid first isolated from beef heart in 1942[1] and found to be present as a small fraction (ca. 10-20\%) of the inner membrane of mitochondria.[2] Cardiolipin is closely associated to the bioenergetic processes.[3, 4] Its specific function arises from its unique dimeric structure containing four unsaturated alkyl chains and an overall net negative charge at neutral $\mathrm{pH}$ (Figure 1).[5] In addition to its key role for maintaining optimal activity of numerous mitochondrial processes such as electron transfer in the respiratory chain, cardiolipin is also involved in the initiation of the cell apoptosis machinery in particular by forming a peroxidase complex with cytochrome-c.[2, 4, 6-14] Indeed a direct relationship between cardiolipin loss and cytochrome- $c$ release into the cytoplasm was identified as an initial step in the pathway to apoptosis.[2] The specific and selective interaction between cardiolipin and cytochrome- $c$ is not well understood and has been assigned to a combination of electrostatic and hydrophobic effects, hydrogen bonding and/or the formation of a cardiolipin/cytochrome- $c$ complex.[2, 6-12, 15-18]

The electrochemical behavior of redox proteins such as cytochrome- $c$, a water-soluble haemoprotein involved in the respiratory chain of mitochondria, has been extensively reported since 1977.[19-22] This model redox protein has often been studied at lipid-modified electrodes because the detection of its electroactivity is seldom possible at bare electrode surfaces even with large scanning potential range.[23-26] This is ascribed to adsorption, structural alteration (denaturation) of the protein at the electrode which prevents or slows down electron transfer.[25, 26] Several surface modification strategies have been used to provide a suitable environment for preserving the native protein structure and promoting electron transfer. In this context, the deposit of a mixed-lipids bilayer onto the electrode surface, mimicking the periplasmic interface of the inner membrane of mitochondria,[3] was demonstrated to be particularly relevant.[25-28] 
Different methods can be successfully employed to form supported lipid bilayers including the Langmuir-Blodgett technique,[26] spin coating of lipid solution,[26, 29] solvent or droplet evaporation [30, 31] or fusion of lipid vesicles at the electrode surface.[32] In most cases, the presence of a fraction of cardiolipin in the supported mixed-lipids bilayer has been demonstrated to be an important factor for the efficient detection of cytochrome- $c$ electroactivity at carbon electrodes.[26, 29, 31] Indeed most of the lipid films investigated to date were mainly composed of phosphatidylcholine associated with a small fraction of cardiolipin. We note that phosphatidylcholine alone is ineffective for the detection of the cytochrome- $c$ electroactivity.[29, 33, 34] To the best of our knowledge however, the electrochemical characterization of a modified electrode with a pure cardiolipin film has never been reported so far except on one occasion with a very high loading of cardiolipin (700 $\mu \mathrm{g} \mathrm{cm}^{-2}$ ) in the absence and in the presence of a cholesterol under-layer.[26] From this literature overview, it is evident that the role of the cardiolipin/cytochrome-c interaction is crucial for electron transfer in the respiratory chain or at an electrode. Nevertheless no previous study focused on the relation between the structure and the organization of supported pure cardiolipin films and the electrochemical behavior of cytochrome- $c$ at the corresponding modified electrodes. In addition, the deposition of a pure cardiolipin film by solvent evaporation is a much more straightforward experimental procedure compared with the preparation of a well-defined supported mixed-lipids bilayer.

We report herein the use of pure cardiolipin (without any other lipids) deposited in optimized surface concentrations by solvent evaporation at the glassy carbon electrode for promoting the electroactivity of cytochrome- $c$ immobilized in its native state. We focus on the organization of an optimized amount of pure cardiolipin on a glassy carbon electrode rather than on the preparation of typical mixed-lipids bilayers. The properties of the optimized pure cardiolipin deposit is investigated with respect to cytochrome- $c$ electroactivity and 
immobilization. First the permeability and compactness of a supported pure cardiolipin film is studied by cyclic voltammetry (CV) and electrochemical impedance spectroscopy (EIS) in the presence of a redox probe (ferri/ferrocyanide) as a function of the amount of lipid deposited. Then, the organization and the thickness of the optimized supported pure cardiolipin film is analyzed by atomic force microscopy (AFM) in liquid conditions and compared with a different pure lipid deposit (1,2-dipalmitoylphosphatidylcholine, DPPC). The optimized pure cardiolipin loading at a glassy carbon electrode is finally tested for cytochrome- $c$ electroactivity with the protein in solution or immobilized onto the lipid deposit.

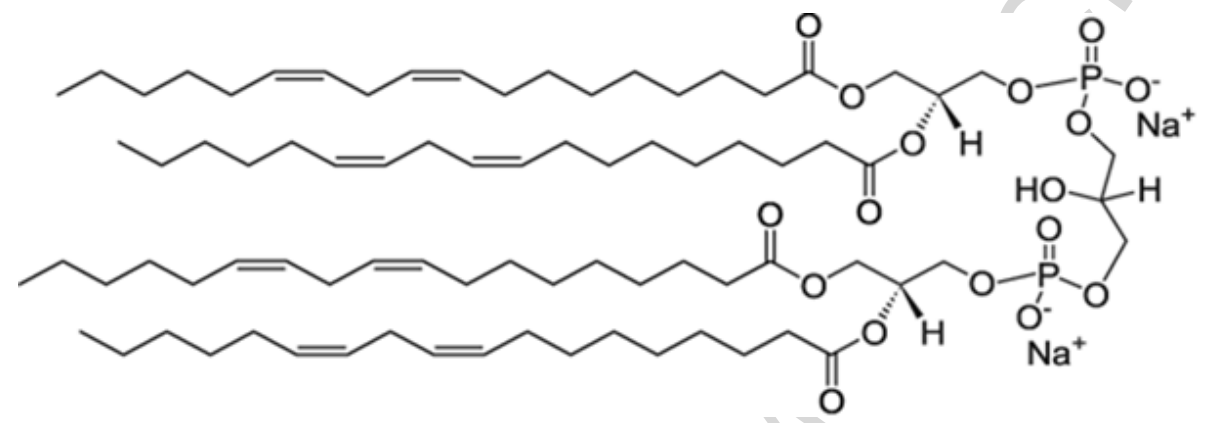

Figure 1. Chemical structure of cardiolipin.

\section{Material and methods}

\subsection{Reagents}

All solutions were prepared with Milli-Q water $\left(18.2 \mathrm{M} \Omega \mathrm{cm}^{-1}\right)$. Cardiolipin solution from bovine heart $(\sim 4.7-5.3 \mathrm{mg} / \mathrm{mL}$ in ethanol, $\geq 97 \%)$ containing $>80 \%$ polyunsaturated fatty acid content, primarily linoleic acid, and cytochrome-c from equine heart ( $\geq 95 \%)$ were obtained from Sigma Aldrich and stored in a freezer $\left(-18^{\circ} \mathrm{C}\right)$. 1,2dipalmitoylphosphatidylcholine (DPPC) lipids were purchased in powder from Avanti Polar Lipids and stored in a freezer. Potassium ferrocyanide trihydrate $(99+\%)$ was purchased from Acros Organics and potassium hexacyanoferrate (III) ( 99\%) from Sigma Aldrich. Sodium hydrogen phosphate anhydrous ACS $(99.0 \% \mathrm{~min})$ and potassium dihydrogen phosphate ACS (99.0\% min) from Alfa Aesar were used to prepare the $10 \mathrm{mM}$ aqueous phosphate buffer $\mathrm{pH}$ 
7. Anhydrous absolute ethanol from Carlo Erba Reagents was used to prepare a cardiolipin solution of $0.2 \mathrm{~g} / \mathrm{L}$ by dilution of the commercial solution and a DPPC solution of $0.3 \mathrm{~g} / \mathrm{L}$.

\subsection{Preparation of cardiolipin-modified electrodes}

The cardiolipin film was obtained by solvent (droplet) evaporation on glassy carbon disk electrodes ( $3 \mathrm{~mm}$ diameter) obtained from BASi. Small volumes between 1 and $10 \mu \mathrm{L}$ of cardiolipin solution diluted at $0.2 \mathrm{~g} / \mathrm{L}$ were deposited on the electrode surface with a micropipette to obtain a total amount of deposited lipids between 0.2 and $2.0 \mu \mathrm{g}$. Ethanol was then left to evaporate under air. After the complete evaporation of ethanol the dry electrode was immediately dipped into the aqueous electrolyte.

\subsection{Electrochemical measurements}

Cyclic voltammetry (CV) and electrochemical impedance spectroscopy (EIS) were performed in a three-electrode cell with a glassy carbon electrode as the working electrode. The working electrode was polished on silicon carbide paper (4000-grid SiC paper, Struers), rinsed with ultra-pure water and sonicated in ultra-pure water for 5 minutes before each cardiolipin deposition or experiment. All potentials are reported versus an $\mathrm{Ag} / \mathrm{AgCl}, \mathrm{KCl} 3 \mathrm{M}$ reference electrode. A platinum wire was used as a counter electrode. Electrochemical experiments were performed at room temperature $\left(21 \pm 3{ }^{\circ} \mathrm{C}\right)$ with an Autolab PGSTAT302N potentiostat/galvanostat (Eco Chemie B.V., the Netherlands) using Nova as the electrochemical software (Metrohm). All solutions were deaerated by bubbling argon for 20 minutes before each measurement. EIS measurements were performed at open circuit potential (OCP: $+0.21 \mathrm{~V}$ ) in the frequency range from $100 \mathrm{kHz}$ down to $50 \mathrm{mHz}$ with a signal amplitude of $10 \mathrm{mV}$, using an equimolar mixture of $\mathrm{K}_{3} \mathrm{Fe}(\mathrm{CN})_{6} / \mathrm{K}_{4} \mathrm{Fe}(\mathrm{CN})_{6}$ as the redox probe (total concentration of $10 \mathrm{mM}$ ). To immobilize cytochrome- $c$ on the deposited cardiolipin 
film, the electrode was immersed in a $0.15 \mathrm{mM}$ cytochrome- $c$ solution and cycled (10 cycles) between +0.3 and $-0.2 \mathrm{~V}$ at a scan rate of $20 \mathrm{mV} \mathrm{s}^{-1}$. Then, the electrode was gently washed with phosphate buffer solution and transferred to a cytochrome-free phosphate buffer electrolyte for cyclic voltammetry experiments.

\subsection{Atomic Force Microscopy}

Pyrolyzed photoresist film (PPF) are used as substrates for imaging the lipid deposits. The procedure followed for PPF preparation is reported in the Supporting Information.[35] The lipid deposit was obtained by solvent evaporation. An appropriate volume of lipid solution diluted at $0.2 \mathrm{~g} / \mathrm{L}$ or $0.3 \mathrm{~g} / \mathrm{L}$ was deposited on the carbon surface with a micropipette to obtain a surface concentration of deposited lipids of approximately $5 \mathrm{nmol} \mathrm{cm}{ }^{-2}$. After the complete evaporation of ethanol, droplets of $10 \mathrm{mM}$ phosphate buffer aqueous electrolyte at pH 7 were added onto the dry modified PPF to keep the lipid film in contact with the liquid phase during AFM measurements. Two types of pure lipid deposits were studied by AFM: cardiolipin (CL) and 1,2-dipalmitoylphosphatidylcholine (DPPC).

AFM height images in liquid were obtained using a Nanoscope 8 Multimode AFM (Bruker) with triangular $\mathrm{Si}_{3} \mathrm{~N}_{4}$ cantilevers (ScanAsyst-Fluid+) with a nominal spring constant of $0.7 \mathrm{~N} \mathrm{~m}^{-1}$. The instrument was equipped with a "J" scanner $(100 \mu \mathrm{m} \times 100 \mu \mathrm{m})$. To minimize the applied force on the sample the set point was continuously adjusted during imaging. Images were acquired with a scan rate of $1 \mathrm{~Hz}$. All images were processed using the Nanoscope 8 software with the ScanAsyst-Fluid mode and were recorded at room temperature $\left(21 \pm 3{ }^{\circ} \mathrm{C}\right)$ 


\section{Results and Discussion}

\subsection{Optimization of the pure cardiolipin deposit}


Figure 2. (Left) Representative CVs recorded at $50 \mathrm{mV} \mathrm{s}^{-1}$ and (Right) Nyquist plots with fit and simulation (equivalent circuit) from electrochemical impedance spectroscopy measurements recorded at OCP on glassy carbon electrodes unmodified (black) and modified by a 3 (green), 7 (orange) and 14 (blue) $\mu \mathrm{g} \mathrm{cm}{ }^{-2}$ cardiolipin deposit in $\mathrm{K}_{4} \mathrm{Fe}(\mathrm{CN})_{6} / \mathrm{K}_{3} \mathrm{Fe}(\mathrm{CN})_{6}$ (both at $5 \mathrm{mM}$ ) with $0.1 \mathrm{M}$ phosphate buffer aqueous electrolyte at $\mathrm{pH} 7$ under inert atmosphere (Ar)

In this section, the optimization of the pure cardiolipin loading onto glassy carbon electrode was performed. In order to optimize and evaluate the permeability and the thickness of supported pure cardiolipin films, three different lipid amounts have been deposited onto glassy carbon corresponding to 3,7 and $14 \mu \mathrm{g} \mathrm{cm}{ }^{-2}$ surface concentrations. These lipid loadings are in the range commonly used for lipid mixture deposition in the literature.[29] These modified electrodes were studied by $\mathrm{CV}$ and EIS in an aqueous redox probe solution of equimolar ferri-/ferrocyanide as presented in Figure 2. This redox probe is particularly sensitive to electrode surface treatment and is especially well-suited for evaluating the effect 
of supported lipid deposits on electron transfer.[28-30, 32, 35-40] CV experiments (Figure 2, Left) show that the electron transfer is partially blocked at the cardiolipin-modified glassy carbon electrodes in comparison to the electrochemically reversible redox system observed at the unmodified glassy carbon electrode. Interestingly, this blocking effect is the highest with the intermediary $7 \mu \mathrm{g} \mathrm{cm}^{-2}$ cardiolipin deposit. EIS measurements (Figure 2, Right) confirm that the charge-transfer resistance is the largest at this modified electrode. The Nyquist plots show a charge-transfer resistance maximized for a cardiolipin deposit of $7 \mu \mathrm{g} \mathrm{cm}^{-2}$ onto glassy carbon electrode while it is lower for the 3 or $14 \mu \mathrm{g} \mathrm{cm}^{-2}$ deposits. This reproducible result (over three independent experiments) suggests that the organization of the cardiolipin film onto glassy carbon is optimized around $7 \mu \mathrm{g} \mathrm{cm}$, with a lower permeability and a more compact structure of the lipid deposit. However, in all cases the lipid deposit does not fully block the redox probe electron transfer probably because of the presence of defects in the supported lipid films and the formation of thick heterogeneous layers onto electrode surface. Note that a similar behavior with the same trend was also observed on cardiolipin-modified gold electrodes (Figure S1) and that CV and EIS experiments with non-negatively charged redox probes such as ferrocenemethanol or ruthenium hexaammine do not show differences between bare and modified electrodes.

A Randles circuit (insert, Figure 2, Right) commonly applied to simulate the EIS data of supported lipid bilayers was used to assess the capacitance and resistance values of cardiolipin deposits.[28, 30, 41-43] In the equivalent circuit, $\mathrm{R}_{\mathrm{s}}$ represents the solution resistance, $R_{m}$ the cardiolipin film resistance, $C_{m}$ the cardiolipin film capacitance, $R_{c t}$ chargetransfer resistance, $\mathrm{C}_{\mathrm{dl}}$ the double-layer capacitance and $\mathrm{Z}_{\mathrm{w}}$ the Warburg element. According to the values of equivalent circuit elements obtained by fitting the experimental data for cardiolipin-modified glassy carbon electrodes with the Nova 2.1 software, the $7 \mu \mathrm{g} \mathrm{cm}^{-2}$ cardiolipin-modified electrode exhibits the highest $R_{m}$ and $R_{c t}$. The $C_{m}$ value is directly 
related to the thickness/packing density of the lipid film and the estimated capacitance per surface unit (electrode area $0.071 \mathrm{~cm}^{2}$ ) are lower than those usually reported for classical supported lipid bilayers.[28, 30, 42, 44, 45] The lowest $C_{m}$ value obtained for the $7 \mu \mathrm{g} \mathrm{cm}^{-2}$ deposit suggests a higher thickness of the supported cardiolipin film coupled to a specific compact structural organization. The experimental EIS data and the simulation (Figure 2, Right) do not show a perfect fit, confirming that the supported lipid film is not organized as a bilayer.

To demonstrate that an optimal surface concentration of pure cardiolipin leads to a more compact and less permeable lipid film on carbon surface with a structure different from a lipid bilayer organization, the aspect and the thickness of lipid deposits in fluid were further probed by atomic force microscopy (AFM) as discussed next.

\subsection{Imaging of the lipid films with liquid AFM measurements}
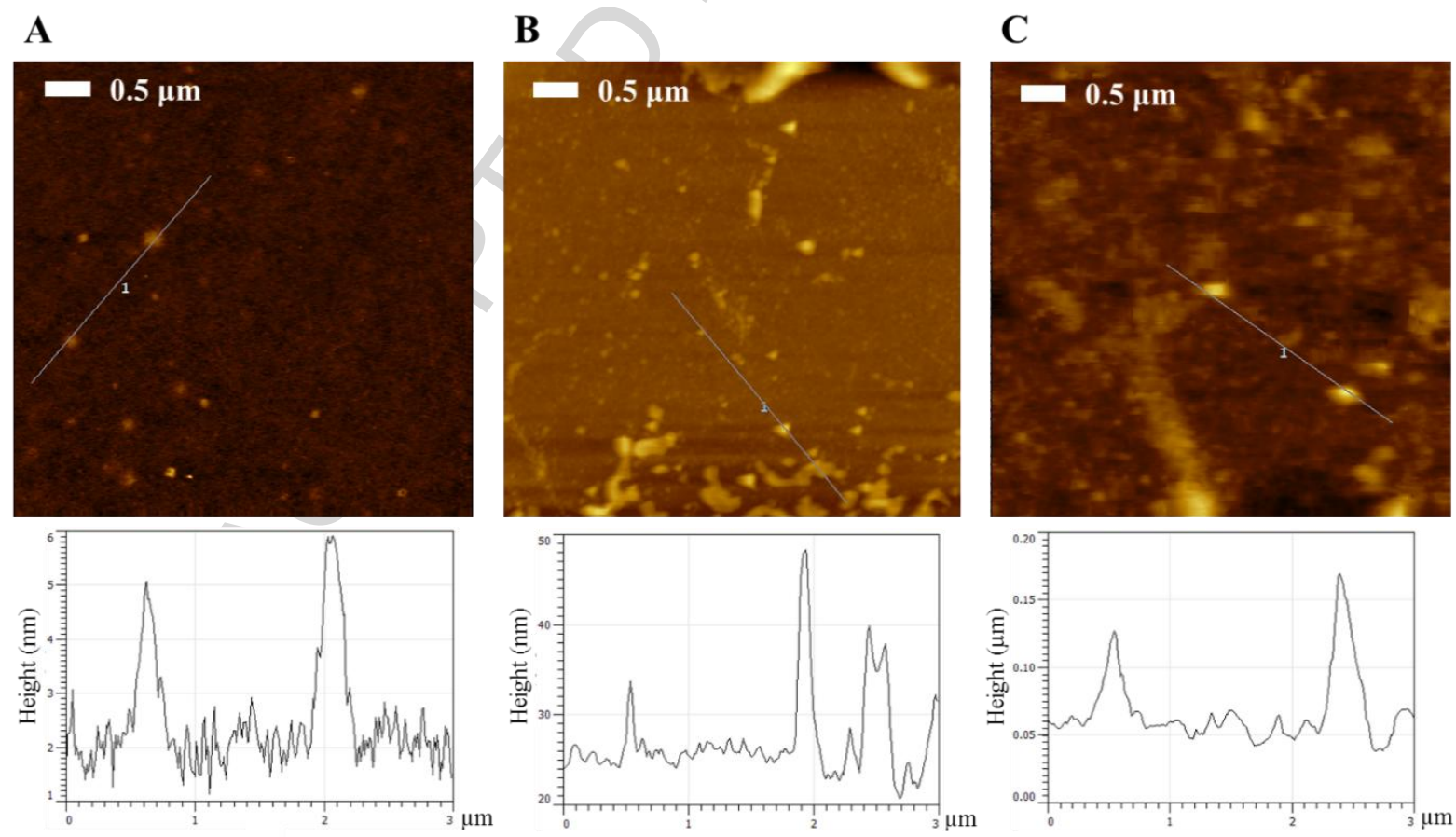
Figure 3. AFM height images $\left(5 \times 5 \mu \mathrm{m}^{2}\right)$ recorded in $10 \mathrm{mM}$ phosphate buffer aqueous electrolyte at $\mathrm{pH} 7$ of a bare PPF (A), a PPF modified by $5 \mathrm{nmol} \mathrm{cm}{ }^{-2}$ DPPC (B) and $5 \mathrm{nmol}$ $\mathrm{cm}^{-2} \mathrm{CL}(\mathrm{C})$ deposit.

For comparing the organization and the thickness of lipid deposits, three PPF samples were observed by atomic force microscopy in liquid (Figure 3): bare PPF surface and PPF surfaces modified by a $5 \mathrm{nmol} \mathrm{cm}$-2 pure lipid deposit of either 1,2-dipalmitoyl-sn-glycero-3phosphocholine (DPPC) or cardiolipin (CL). PPF samples are chosen because their structure is very close to that of glassy carbon, while exhibiting a very smooth surface suitable for AFM measurements. The lipid surface concentration deposited on PPF substrates corresponds to the optimized cardiolipin loading previously reached on glassy carbon electrode $(\sim 7 \mu \mathrm{g}$ $\mathrm{cm}^{-2}$ ). The bare PPF sample image is presented in Figure 3A where the approximate height is evaluated at $3 \pm 1 \mathrm{~nm}$ and the roughness average is estimated at $0.3 \mathrm{~nm}$, in agreement with this nearly atomically smooth carbon surface.[46] DPPC is a typical lipid used to form supported lipid bilayer, it bears two alkyl chains and is zwitterionic and neutral at $\mathrm{pH} 7$. The main reason to use this lipid for comparison with cardiolipin is that DPPC phase transition temperature $\left(40{ }^{\circ} \mathrm{C}\right)$ is comparable to that of cardiolipin $\left(60^{\circ} \mathrm{C}\right)$ and hence the lipids are in the same phase in our experimental conditions at room temperature. After the deposition of DPPC on PPF (Figure 3B), a heterogeneous film is observed with a height of $9 \pm 4 \mathrm{~nm}$ and a roughness average of $1 \mathrm{~nm}$. Several lipid agglomerates appear on PPF sample modified by the DPPC deposit with a random distribution. In addition the PPF surface seems only partially covered by the DPPC lipid deposit. This heterogeneous surface organization shows that the solvent evaporation method is not appropriate for the preparation of uniform supported lipid bilayers. Deposition of pure cardiolipin on PPF yields a lipid film with a higher thickness and surface coverage and the presence of defects (Figure 3C). Indeed, the approximate height of 
cardiolipin deposit is estimated at $30 \pm 10 \mathrm{~nm}$ for a roughness average at $4 \mathrm{~nm}$. Despite the presence of defects and a high roughness, the cardiolipin deposit seems to completely cover the PPF surface. This huge difference between DPPC and cardiolipin deposits definitely confirms the absence of a lipid bilayer in both cases and suggests an organization of the cardiolipin film more voluminous and less even than classical supported lipid layers. Finally, these AFM images demonstrate that a small amount of cardiolipin deposited onto carbon surface leads to the formation of a thick and rough film able to partially block the electron transfer of a redox probe as discussed above. In order to evaluate the impact of the pure cardiolipin deposit toward the electrochemical activity of cytochrome-c, cyclic voltammetry measurements were recorded at a cardiolipin-modified glassy carbon electrode.

\subsection{Cytochrome-c electroactivity in solution and immobilized onto the electrode}

In this section, two efficient methods easy to perform are presented for studying the cytochrome- $c$ electroactivity at a glassy carbon electrode modified by the optimized supported cardiolipin deposit. The protein is first detected in solution (Figure 4A). Then, by potential cycling in the cytochrome $c$ solution, the redox protein is immobilized on the cardiolipinmodified glassy carbon electrode. Finally the electrochemical response of the immobilized cytochrome $c$ can be observed in an aqueous protein-free electrolyte (Figure 4B). 

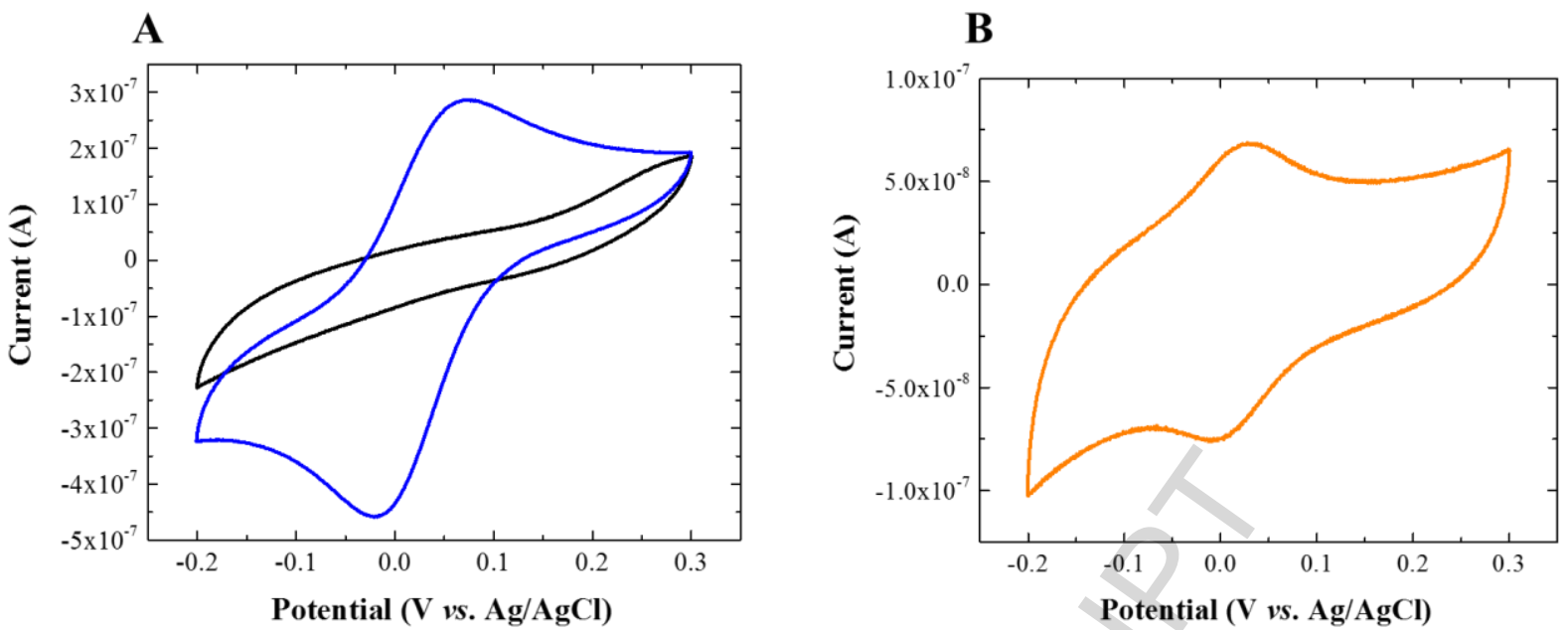

Figure 4. $\mathrm{CV}$ recorded at $20 \mathrm{mV} \mathrm{s}^{-1}$ on glassy carbon electrodes in $10 \mathrm{mM}$ phosphate buffer

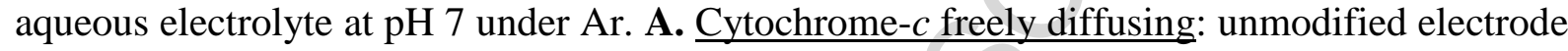
(black) and electrode modified with a $7 \mu \mathrm{g} \mathrm{cm}^{-2}$ cardiolipin deposit (blue) in a $0.15 \mathrm{mM}$ cytochrome- $c$ solution. B. Immobilized cytochrome- $c$ (orange): electrode modified with a 7 $\mu \mathrm{g} \mathrm{cm}^{-2}$ cardiolipin deposit, first cycled ten times in a $0.15 \mathrm{mM}$ cytochrome- $c$ solution for protein immobilization and then transferred in a cytochrome-free electrolyte.

In Figure 4A, no redox system is detected on an unmodified glassy carbon electrode in a cytochrome- $c$ solution in the scanned potential range because of the protein denaturation or unfavorable orientation.[26, 27] As expected, the same result is obtained at the phosphatidylcholine-modified glassy carbon electrode (Figure S2). In contrast, at the $7 \mu \mathrm{g} \mathrm{cm}^{-}$ ${ }^{2}$ cardiolipin modified glassy carbon electrode, cytochrome- $c$ in solution displays a welldefined quasi-reversible $\left(\Delta E_{p}=90 \pm 4 \mathrm{mV}\right)$ redox system at $0.025 \mathrm{~V}$. From the linear relationship of the oxidative peak currents versus the square root of the scan rate, the diffusion coefficient was estimated to $6.4 \times 10^{-7} \mathrm{~cm}^{2} \mathrm{~s}^{-1}$, by applying the analytical equation for a quasireversible monoelectronic electron transfer (vide infra) (Figure S3A, see SI).[47] This value is in good agreement with those reported in literature for cytochrome-c in solution.[20, 48-50] The apparent kinetic rate constant has been estimated through numerical simulations of the 
cyclic voltammograms using the KISSA software.[51, 52] The kinetic rate constant $k_{\mathrm{s}}$ of cytochrome- $c$ in solution at a cardiolipin-modified glassy carbon electrode was determined as $1.0( \pm 0.5) \times 10^{-3} \mathrm{~cm} \mathrm{~s}^{-1}$. This value is consistent with the one reported previously $\left(2.0 \times 10^{-3}\right.$ $\left.\mathrm{cm} \mathrm{s}^{-1}\right)$ at a glassy carbon electrode modified with a high loading of pure cardiolipin $(700 \mu \mathrm{g}$ $\mathrm{cm}^{-2}$ ).[24] The estimated kinetic rate constant also falls in the range reported for other redox proteins in solution.[53] This result shows that only a small amount of pure cardiolipin (7 $\mu \mathrm{g}$ $\mathrm{cm}^{-2}$ ) simply deposited by solvent evaporation is sufficient for promoting the direct electron transfer of cytochrome- $c$ in solution as a stable and reproducible redox signal at a modified glassy carbon electrode.

A simple and efficient strategy to immobilize cytochrome- $c$ on the lipid modified electrode consists in performing recurrent potential cycles (here ten cycles, until a steady state current is reached, Figure S4A) in a cytochrome- $c$ solution and then to transfer the modified electrode in a protein-free phosphate buffer where the electroactivity of the immobilized protein can be studied.[26, 29] In Figure 4B, the cyclic voltammogram recorded in a proteinfree aqueous electrolyte on cardiolipin-modified glassy carbon electrode and previously cycled in cytochrome- $c$ solution displays a redox system at $+0.012 \mathrm{~V}$ assigned to cytochrome$c$ adsorbed or aggregated to the supported cardiolipin film. The peak-to-peak potential separation $\left(\Delta E_{p}=30 \pm 2 \mathrm{mV}\right.$ at $0.02 \mathrm{~V} \mathrm{~s}^{-1}, 45 \pm 2 \mathrm{mV}$ at $\left.0.05 \mathrm{~V} . \mathrm{s}^{-1}\right)$ is consistent with the immobilization of cytochrome- $c$ at the lipid-modified electrode as reported in other works involving cardiolipin based films on carbon surface.[26, 41] For instance, the electrochemical signal of cytochrome- $c$ immobilized at a glassy carbon electrode coated with a mixture of cholesterol/cardiolipin exhibits a peak-to-peak potential, $\Delta E_{p}=80 \mathrm{mV}$, at $0.05 \mathrm{~V} \mathrm{~s}^{-1}$.[26] This corresponds to an electrochemically quasi-reversible electron transfer reaction. In contrast, at a well-organized monolayer of 11-mercaptoundecanoic acid on a gold the immobilization of cytochrome-c led to an electrochemically reversible system as shown by a peak-to-peak 
separation equal to $18 \mathrm{mV}$ at $0.2 \mathrm{~V} \mathrm{~s}^{-1}$.[54] The oxidative peak currents are linearly proportional to the scan rates up to $100 \mathrm{mV} \mathrm{s}^{-1}$, consistent with an adsorption-controlled process (Figure S3B).[32] We also note that the redox potential of cytochrome-c in solution and immobilized onto modified glassy carbon electrode are close $(+25$ and $+12 \mathrm{mV}$ vs. $\mathrm{Ag} / \mathrm{AgCl}, \mathrm{KCl} 3 \mathrm{M})$. This suggests that the protein is present in its native state onto the surface and not as a cardiolipin/cytochrome- $c$ complex that would yield a significant potential cathodic shift of the protein redox potential (> $0.3 \mathrm{~V}) .[16,29,55]$ Indeed, cyclic voltammograms recorded at different scan rates and in a wider potential window (from -0.6 to $+0.7 \mathrm{~V}$ ) showed that the immobilized cytochrome- $c$ is not denatured because of the absence of an additional reduction peak at more negative potential (Figure S3).[16-18, 22] A slight desorption of the immobilized protein could be observed after ten potential cycles between $+0.3 \mathrm{~V}$ and $-0.2 \mathrm{~V}$, which led to about a $10 \%$ decrease of the redox signal (Figure S4B).[1618] This indicates a better stability of the immobilized cytochrome- $c$ at the glassy carbon electrode modified with a small amount of cardiolipin $\left(7 \mu \mathrm{g} \mathrm{cm}^{-2}\right)$ in comparison with a previous study with a high cardiolipin lipid loading at the electrode $\left(\sim 700 \mu \mathrm{g} \mathrm{cm}^{-2}\right)$.[26] Indeed, in this latter work, the decrease of the protein redox signal was approximately $30 \%$ after three cyclic voltammograms. Thus, the immobilization of cytochrome- $c$ onto the modified glassy carbon electrode surface is assigned to specific and selective interactions between cytochrome-c and cardiolipin as documented in the literature.[2, 6-9, 15] The electron transfer rate constant $k_{\mathrm{ET}}$ of cytochrome-c immobilized on cardiolipin-modified glassy carbon electrode has been estimated through numerical simulations [51, 52] of the cyclic voltammograms and amounts to $1.5 \pm 0.5 \mathrm{~s}^{-1}$. This value is slightly lower than those reported previously for immobilized cytochrome- $c$ at cardiolipin/phosphatidylcholinemodified glassy carbon electrodes $\left(8\right.$ to $\left.30 \mathrm{~s}^{-1}\right)$ where the lipid deposit is characteristic to a bilayer of $2 \mathrm{~nm}$ thick,[29] probably because of the higher thickness of the pure cardiolipin 
deposit $(\sim 30 \mathrm{~nm})$. It is also significantly lower than those reported for cytochrome- $c$ covalently attached to modified gold electrodes via a mercaptoheptanoic acid self-assembled monolayer (200 to $250 \mathrm{~s}^{-1}$ )[16] but close to those reported for cytochrome- $c$ covalently attached to modified gold electrodes via a mercaptohexadecanoic acid self-assembled monolayer $\left(3 \mathrm{~s}^{-1}\right)$.[56] In addition, the surface coverage of the immobilized electroactive cytochrome- $c$ estimated from faradic charge integration of the cyclic voltammogram is ca. 8 pmol cm $\mathrm{cm}^{-2}$ and corresponds to ca. $50 \%$ of a theoretical protein monolayer. This is in agreement with previous studies using various modified glassy carbon or gold electrodes (self-assembled monolayers, lipid mono/bilayers).[16, 29, 57] Moreover, the estimated electron transfer rate $k_{E T}$ for cytochrome-c immobilized at the rather thick cardiolipin deposit $\left(1.5 \pm 0.5 \mathrm{~s}^{-1}\right)$ is close to that reported for cytochrome- $c$ covalently bound to a self-assembled monolayer of 16-mercaptohexadecanoic acid onto gold surfaces (1 to $\left.3 \mathrm{~s}^{-1}\right)$.[56] This work demonstrates that a supported bilayer based on mixed-lipids and mimicking the inner membrane of mitochondria is not strictly required for immobilizing the redox protein in its native state and for promoting direct electron transfer between cytochrome-c and an electrode. The deposition of pure cardiolipin by solvent evaporation onto glassy carbon surface is an easy, low cost and efficient method for promoting and keeping intact the electrochemical properties of cytochrome-c both in solution and adsorbed on surface.

\section{Conclusions}

In summary, pure cardiolipin deposited onto glassy carbon electrode surface has been optimized for maximum compactness at low lipid loading. Cyclic voltammetry and electrochemical impedance spectroscopy measurements recorded in ferri/ferrocyanide solution have demonstrated that the most compact and the less permeable cardiolipin film was reached for a $7 \mu \mathrm{g} \mathrm{cm}^{-2}$ deposit on glassy carbon. AFM experiments in liquid phase have been 
performed and have shown the formation of a thick and rough cardiolipin film with a height of the order of $30 \pm 10 \mathrm{~nm}$ on the PPF sample. The optimized supported cardiolipin deposit at glassy carbon was used to investigate cytochrome-c electroactivity either in solution or immobilized at the modified surface. With cytochrome- $c$ in solution, cyclic voltammetry experiments showed that the pure cardiolipin deposit made by simple solvent evaporation is necessary and sufficient for promoting the electron-transfer between the redox protein and the modified electrode. In addition, recurrent cycles in the cytochrome- $c$ solution led to the immobilization of the protein in its native state into the lipid film with retention of its electroactivity. The pure cardiolipin deposit discussed here is not an authentic biomimetic system because cardiolipin is usually only a minor component of biological membranes and the deposit studied in this work is not organized as a bilayer. This work nevertheless demonstrates that cytochrome- $c$ can be anchored to pure cardiolipin-modified electrode surface and detected electrochemically in its native state. It also suggests that other enzymes could be immobilized in this matrix for sensing or biofuel cell applications.

\section{Corresponding Authors}

frederic.barriere@univ-rennes1.fr

estelle.lebegue@univ-rennes1.fr

\section{Competing Financial Interest}

The authors declare no competing financial interest.

\section{Acknowledgements}

The authors thank ANR-15-CE05-0003 bioWATTS project for support. Estelle Lebègue is supported by a Marie Skłodowska Curie Individual Fellowship. Prof. I. Svir, C. Amatore and 
O. Klymenko are warmly thanked for providing the KISSA Software. The authors are grateful to the 2CBioMIF facilities for the AFM experiments (ScanMAT, UMS 2001 CNRS University of Rennes 1, supported by Région Bretagne and European Union CPER-FEDER 2007-2014).

\section{References}

[1] M.C. Pangborn, ISOLATION AND PURIFICATION OF A SEROLOGICALLY ACTIVE PHOSPHOLIPID FROM BEEF HEART, Journal of Biological Chemistry 143(1) (1942) 247-256.

[2] J.B. McMillin, W. Dowhan, Cardiolipin and apoptosis, Biochimica et Biophysica Acta (BBA) - Molecular and Cell Biology of Lipids 1585(2-3) (2002) 97-107.

[3] G. Daum, Lipids of mitochondria, Biochimica et Biophysica Acta (BBA) - Reviews on Biomembranes 822(1) (1985) 1-42.

[4] G. Paradies, V. Paradies, V. De Benedictis, F.M. Ruggiero, G. Petrosillo, Functional role of cardiolipin in mitochondrial bioenergetics, Biochimica et Biophysica Acta (BBA) Bioenergetics 1837(4) (2014) 408-417.

[5] P.V. Ioannou, B.T. Golding, Cardiolipins: Their Chemistry and Biochemistry, Progress in Lipid Research 17(3) (1979) 279-318.

[6] F. Sinibaldi, B.D. Howes, M.C. Piro, F. Polticelli, C. Bombelli, T. Ferri, M. Coletta, G. Smulevich, R. Santucci, Extended cardiolipin anchorage to cytochrome c: a model for protein-mitochondrial membrane binding, JBIC Journal of Biological Inorganic Chemistry 15(5) (2010) 689-700.

[7] E.K.J. Tuominen, C.J.A. Wallace, P.K.J. Kinnunen, Phospholipid-Cytochrome c Interaction: EVIDENCE FOR THE EXTENDED LIPID ANCHORAGE, Journal of Biological Chemistry 277(11) (2002) 8822-8826.

[8] J. Pan, X. Cheng, M. Sharp, C.-S. Ho, N. Khadka, J. Katsaras, Structural and mechanical properties of cardiolipin lipid bilayers determined using neutron spin echo, small angle neutron and X-ray scattering, and molecular dynamics simulations, Soft Matter 11(1) (2015) $130-138$.

[9] F. Sinibaldi, L. Milazzo, B.D. Howes, M.C. Piro, L. Fiorucci, F. Polticelli, P. Ascenzi, M. Coletta, G. Smulevich, R. Santucci, The key role played by charge in the interaction of cytochrome c with cardiolipin, JBIC Journal of Biological Inorganic Chemistry 22(1) (2017) 19-29.

[10] J.M. Bradley, G. Silkstone, M.T. Wilson, M.R. Cheesman, J.N. Butt, Probing a Complex of Cytochrome c and Cardiolipin by Magnetic Circular Dichroism Spectroscopy: Implications 
for the Initial Events in Apoptosis, Journal of the American Chemical Society 133(49) (2011) 19676-19679.

[11] J. Muenzner, E.V. Pletneva, Structural transformations of cytochrome c upon interaction with cardiolipin, Chemistry and Physics of Lipids 179 (2014) 57-63.

[12] L. Milazzo, L. Tognaccini, B.D. Howes, F. Sinibaldi, M.C. Piro, M. Fittipaldi, M.C. Baratto, R. Pogni, R. Santucci, G. Smulevich, Unravelling the Non-Native Low-Spin State of the Cytochrome c-Cardiolipin Complex: Evidence of the Formation of a His-Ligated Species Only, Biochemistry 56(13) (2017) 1887-1898.

[13] V.E. Kagan, H.A. Bayır, N.A. Belikova, O. Kapralov, Y.Y. Tyurina, V.A. Tyurin, J. Jiang, D.A. Stoyanovsky, P. Wipf, P.M. Kochanek, J.S. Greenberger, B. Pitt, A.A. Shvedova, G. Borisenko, Cytochrome c/cardiolipin relations in mitochondria: a kiss of death, Free Radical Biology and Medicine 46(11) (2009) 1439-1453.

[14] Z.T. Schug, E. Gottlieb, Cardiolipin acts as a mitochondrial signalling platform to launch apoptosis, Biochimica et Biophysica Acta (BBA) - Biomembranes 1788(10) (2009) 20222031.

[15] J.P. Kitt, D.A. Bryce, S.D. Minteer, J.M. Harris, Raman Spectroscopy Reveals Selective Interactions of Cytochrome c with Cardiolipin That Correlate with Membrane Permeability, Journal of the American Chemical Society 139(10) (2017) 3851-3860.

[16] L.V. Basova, I.V. Kurnikov, L. Wang, V.B. Ritov, N.A. Belikova, I.I. Vlasova, A.A. Pacheco, D.E. Winnica, J. Peterson, H. Bayir, D.H. Waldeck, V.E. Kagan, Cardiolipin Switch in Mitochondria: Shutting off the Reduction of Cytochrome $\mathrm{c}$ and Turning on the Peroxidase Activity, Biochemistry 46(11) (2007) 3423-3434.

[17] A. Ranieri, G. Di Rocco, D. Millo, G. Battistuzzi, C.A. Bortolotti, L. Lancellotti, M. Borsari, M. Sola, Thermodynamics and kinetics of reduction and species conversion at a hydrophobic surface for mitochondrial cytochromes $\mathrm{c}$ and their cardiolipin adducts, Electrochimica Acta 176 (2015) 1019-1028.

[18] A. Ranieri, D. Millo, G. Di Rocco, G. Battistuzzi, C.A. Bortolotti, M. Borsari, M. Sola, Immobilized cytochrome $\mathrm{c}$ bound to cardiolipin exhibits peculiar oxidation state-dependent axial heme ligation and catalytically reduces dioxygen, JBIC Journal of Biological Inorganic Chemistry 20(3) (2015) 531-540.

[19] M.J. Eddowes, H.A.O. Hill, Novel method for the investigation of the electrochemistry of metalloproteins: cytochrome c, Journal of the Chemical Society, Chemical Communications (21) (1977) 771b-772.

[20] M.J. Eddowes, H.A.O. Hill, Electrochemistry of horse heart cytochrome c, Journal of the American Chemical Society 101(16) (1979) 4461-4464.

[21] A. Bhambhani, S. Chah, E.G. Hvastkovs, G.C. Jensen, J.F. Rusling, R.N. Zare, C.V. Kumar, Folding Control and Unfolding Free Energy of Yeast Iso-1-cytochrome c Bound to Layered Zirconium Phosphate Materials Monitored by Surface Plasmon Resonance, The Journal of Physical Chemistry B 112(30) (2008) 9201-9208. 
[22] D. Novak, M. Mojovic, A. Pavicevic, M. Zatloukalova, L. Hernychova, M. Bartosik, J. Vacek, Electrochemistry and electron paramagnetic resonance spectroscopy of cytochrome c and its heme-disrupted analogs, Bioelectrochemistry 119(Supplement C) (2018) 136-141.

[23] S. Chao, J.L. Robbins, M.S. Wrighton, A new ferrocenophane surface derivatizing reagent for the preparation of nearly reversible electrodes for horse heart ferri/ferrocytochrome c: 2,3,4,5-tetramethyl-1-[(dichlorosilyl)methyl][2]ferrocenophane, Journal of the American Chemical Society 105(2) (1983) 181-188.

[24] Z. Salamon, G. Tollin, Interfacial electrochemistry of cytochrome c at a lipid bilayer modified electrode: effect of incorporation of negative charges into the bilayer on cyclic voltammetric parameters, Journal of Electroanalytical Chemistry and Interfacial Electrochemistry 321(2) (1991) 321-334.

[25] Z. Salamon, J.T. Hazzard, G. Tollin, Direct measurement of cyclic current-voltage responses of integral membrane proteins at a self-assembled lipid-bilayer-modified electrode: cytochrome $\mathrm{f}$ and cytochrome c oxidase, Proceedings of the National Academy of Sciences 90(14) (1993) 6420-6423.

[26] H. Park, J.-S. Park, Y.-B. Shim, Electrochemical and in situ UV-visible spectroscopic behavior of cytochrome $\mathrm{c}$ at a cardiolipin-modified electrode, Journal of Electroanalytical Chemistry 514(1-2) (2001) 67-74.

[27] J. Wang, M. Li, Z. Shi, N. Li, Z. Gu, Direct Electrochemistry of Cytochrome c at a Glassy Carbon Electrode Modified with Single-Wall Carbon Nanotubes, Analytical Chemistry 74(9) (2002) 1993-1997.

[28] X. Lu, T. Liao, L. Ding, X. Liu, Y. Zhang, Y. Cheng, J. Du, Interaction of quercetin with supported bilayer lipid membranes on glassy carbon electrode, International Journal of Electrochemical Science 3(7) (2008) 797-805.

[29] A. Perhirin, E. Kraffe, Y. Marty, F. Quentel, P. Elies, F. Gloaguen, Electrochemistry of cytochrome $\mathrm{c}$ immobilized on cardiolipin-modified electrodes: A probe for protein-lipid interactions, Biochimica et Biophysica Acta (BBA) - General Subjects 1830(3) (2013) 27982803.

[30] Z. Wu, B. Wang, Z. Cheng, X. Yang, S. Dong, E. Wang, A facile approach to immobilize protein for biosensor: self-assembled supported bilayer lipid membranes on glassy carbon electrode, Biosensors and Bioelectronics 16(1-2) (2001) 47-52.

[31] Y. Huang, L. Liu, C. Shi, J. Huang, G. Li, Electrochemical analysis of the effect of Ca2+ on cardiolipin-cytochrome c interaction, Biochimica et Biophysica Acta (BBA) - General Subjects 1760(12) (2006) 1827-1830.

[32] L. Liu, L. Zeng, L. Wu, X. Jiang, Label-Free Surface-Enhanced Infrared Spectroelectrochemistry Studies the Interaction of Cytochrome c with Cardiolipin-Containing Membranes, The Journal of Physical Chemistry C 119(8) (2015) 3990-3999. 
[33] K.-H. Shin, T. Fujiwara, H. Akutsu, Modulation of the specific interaction of cardiolipin with cytochrome $\mathrm{c}$ by zwitterionic phospholipids in binary mixed bilayers; a $2 \mathrm{H}$ and $31 \mathrm{P}$ NMR study, Journal of Molecular Structure 355(1) (1995) 47-53.

[34] S. Boussaad, L. Dziri, R. Arechabaleta, N.J. Tao, R.M. Leblanc, Electron-Transfer Properties of Cytochrome c Langmuir-Blodgett Films and Interactions of Cytochrome $\mathrm{c}$ with Lipids, Langmuir 14(21) (1998) 6215-6219.

[35] P.A. Brooksby, A.J. Downard, Electrochemical and Atomic Force Microscopy Study of Carbon Surface Modification via Diazonium Reduction in Aqueous and Acetonitrile Solutions, Langmuir 20(12) (2004) 5038-5045.

[36] P. Chen, R.L. McCreery, Control of Electron Transfer Kinetics at Glassy Carbon Electrodes by Specific Surface Modification, Analytical Chemistry 68(22) (1996) 3958-3965.

[37] S. Baranton, D. Belanger, Electrochemical Derivatization of Carbon Surface by Reduction of in Situ Generated Diazonium Cations, Journal of Physical Chemistry B 109 (2005) 24401-24410.

[38] S. Chen, K. Huang, Ion-Induced Interfacial Dynamics of Phospholipid Monolayers, Analytical Chemistry 72(13) (2000) 2949-2956.

[39] M. Twardowski, R.G. Nuzzo, Phase Dependent Electrochemical Properties of Polar SelfAssembled Monolayers (SAMs) Modified via the Fusion of Mixed Phospholipid Vesicles, Langmuir 20(1) (2004) 175-180.

[40] K.P. Divya, V. Dharuman, Supported binary liposome vesicle-gold nanoparticle for enhanced label free DNA and protein sensing, Biosensors and Bioelectronics 95(Supplement C) (2017) 168-173.

[41] K.-S. Lee, M.-S. Won, H.-B. Noh, Y.-B. Shim, Triggering the redox reaction of cytochrome $\mathrm{c}$ on a biomimetic layer and elimination of interferences for NADH detection, Biomaterials 31(30) (2010) 7827-7835.

[42] F. Bordi, C. Cametti, A. Gliozzi, Impedance measurements of self-assembled lipid bilayer membranes on the tip of an electrode, Bioelectrochemistry 57(1) (2002) 39-46.

[43] M. Legiň, G. Laputková, J. Sabo, L. Vojčíková, Impedance spectroscopy of bilayer lipid membranes self-assembled on agar support - Interaction with HDL, Physiological Research 56(SUPPL. 1) (2007) S85-S91.

[44] A.L. Plant, Supported Hybrid Bilayer Membranes as Rugged Cell Membrane Mimics, Langmuir 15(15) (1999) 5128-5135.

[45] R. Fettiplace, D.M. Andrews, D.A. Haydon, The thickness, composition and structure of some lipid bilayers and natural membranes, The Journal of Membrane Biology 5(3) (1971) 277-296.

[46] S. Ranganathan, R.L. McCreery, Electroanalytical Performance of Carbon Films with Near-Atomic Flatness, Analytical Chemistry 73(5) (2001) 893-900. 
[47] J.-M. Savéant, Single Electron Transfer at an Electrode, Elements of Molecular and Biomolecular Electrochemistry, John Wiley \& Sons, Inc.2006, pp. 1-77.

[48] W.R. Hagen, Direct electron transfer of redox proteins at the bare glassy carbon electrode, European Journal of Biochemistry 182(3) (1989) 523-530.

[49] T.D. Dolidze, D.E. Khoshtariya, D.H. Waldeck, J. Macyk, R. van Eldik, Positive Activation Volume for a Cytochrome C Electrode Process: Evidence for a "Protein Friction" Mechanism from High-Pressure Studies, The Journal of Physical Chemistry B 107(29) (2003) 7172-7179.

[50] Y. Dai, D.A. Proshlyakov, G.M. Swain, Effects of Film Morphology and Surface Chemistry on the Direct Electrochemistry of Cytochrome c at Boron-Doped Diamond Electrodes, Electrochimica acta 197 (2016) 129-138.

[51] C. Amatore, O. Klymenko, I. Svir, A new strategy for simulation of electrochemical mechanisms involving acute reaction fronts in solution: Application to model mechanisms, Electrochemistry Communications 12(9) (2010) 1165-1169.

[52] KISSAGroup web site address: http://www.kissagroup.com/.

[53] G. Loget, S. Chevance, C. Poriel, G. Simonneaux, C. Lagrost, J. Rault-Berthelot, Direct Electron Transfer of Hemoglobin and Myoglobin at the Bare Glassy Carbon Electrode in an Aqueous BMI.BF4 Ionic-Liquid Mixture, ChemPhysChem 12(2) (2011) 411-418.

[54] D. Millo, A. Ranieri, P. Gross, H.K. Ly, M. Borsari, P. Hildebrandt, G.J.L. Wuite, C. Gooijer, G.v.d. Zwan, Electrochemical Response of Cytochrome c Immobilized on Smooth and Roughened Silver and Gold Surfaces Chemically Modified with 11Mercaptounodecanoic Acid, The Journal of Physical Chemistry C 113(7) (2009) 2861-2866.

[55] M.A. Marchenkova, Y.A. Dyakova, E.Y. Tereschenko, M.V. Kovalchuk, Y.A. Vladimirov, Cytochrome c Complexes with Cardiolipin Monolayer Formed under Different Surface Pressure, Langmuir 31(45) (2015) 12426-12436.

[56] H. Yue, D.H. Waldeck, J. Petrović, R.A. Clark, The Effect of Ionic Strength on the Electron-Transfer Rate of Surface Immobilized Cytochrome c, The Journal of Physical Chemistry B 110(10) (2006) 5062-5072.

[57] M.T. de Groot, T.H. Evers, M. Merkx, M.T.M. Koper, Electron Transfer and Ligand Binding to Cytochrome c Immobilized on Self-Assembled Monolayers, Langmuir 23(2) (2007) 729-736. 


\section{Table of Content Graphical Abstract}

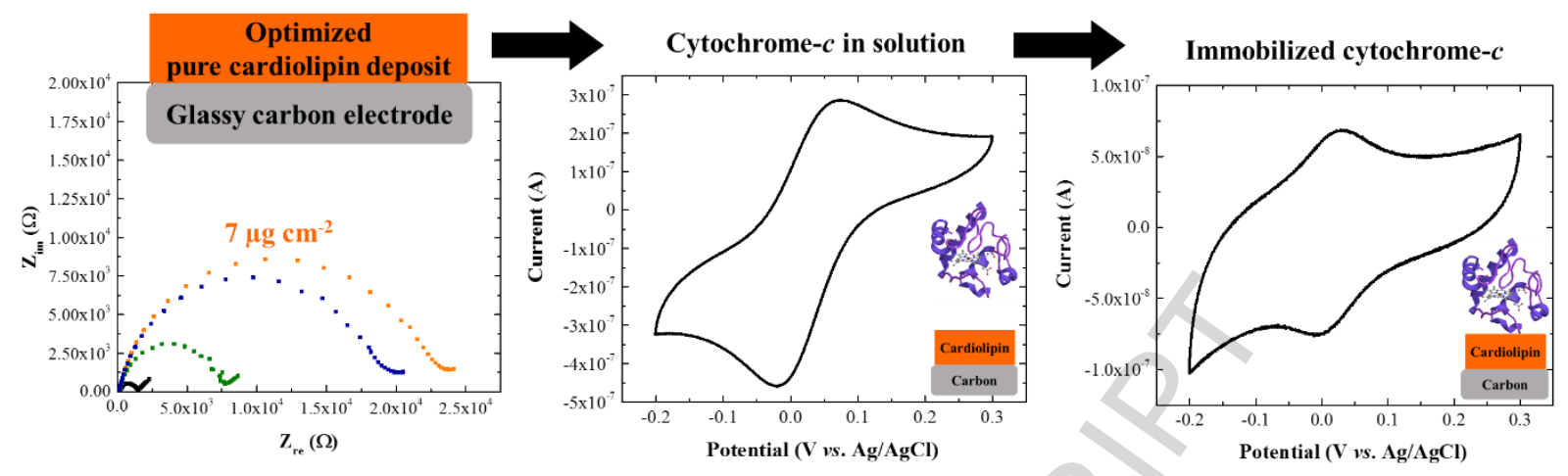




\section{Highlights}

- Cardiolipin-modified glassy carbon electrode is optimized for a $7 \mu \mathrm{g} \mathrm{cm}^{-2}$ deposit.

- Thick surface lipid film of $30 \pm 10 \mathrm{~nm}$ is obtained for cardiolipin deposit on PPF.

- Cardiolipin deposit is sufficient for promoting the cytochrome- $c$ electroactivity.

- Cardiolipin deposit is efficient for immobilizing cytochrome- $c$ in its native state.

- Cardiolipin film is suited to detect cytochrome- $c$ both in solution and on surface. 